\title{
Reabilitação dentogengival em paciente geriátrico acompanhamento de dois anos
}

\author{
Oral rehabilitation with mediate implants in a geriatric patient: Two-year follow-up \\ Rehabilitación oral con implantes mediatos en un paciente geriátrico: Seguimiento a dos años
}

Annika Ingrid Maria Soderberg Campos ORCID: https://orcid.org/0000-0002-6299-3509

Universidade Santo Amaro, Brasil E-mail cdannika@uol.com.br Aziz Semi Chami ORCID: https://orcid.org/0000-0003-4579-1494 Universidade Santo Amaro, Brasil E-mail: azizchami@gmail.com

Angélica Castro Pimentel ORCID: https://orcid.org/0000-0002-6317-6439 Universidade Santo Amaro, Brasil E-mail: acpimentel@prof.unisa.br Wilson Roberto Sendyk ORCID: https://orcid.org/0000-0002-3742-1330 Universidade Santo Amaro, Brasil E-mail: wsendyk@prof.unisa.br

Débora Serrano de Macedo

ORCID: https://orcid.org/0000-0003-2087-8614 Universidade Santo Amaro, Brasil

E-mail: deboraserrano.m@gmail.com Heloisa Fonseca Marão

ORCID: https://orcid.org/0000-0002-6128-8576 Universidade Santo Amaro, Brasil E-mail: hfmarao@prof.unisa.br

\begin{abstract}
Resumo
O objetivo deste estudo foi avaliar a taxa de sobrevivência e sucesso dos implantes em um paciente idoso e investigar as condições funcionais e nutricionais do paciente após o tratamento de reabilitação com uma prótese do tipo protocolo, utilizando carga mediata. A extração dos dentes 33 a 44 foram realizadas e após seis meses foram colocados cinco implantes Cone-Morse de 3,5 x $11 \mathrm{~mm}$. A instalação da prótese foi concluída após 6 meses de osseointegração e o acompanhamento foi realizado por dois anos. Não houve perda de implantes, sinais clínicos de inflamação ou presença de perda óssea ao redor dos implantes, e os componentes protéticos estavam perfeitamente adaptados. Assim, o protocolo de carregamento mediado em pacientes muito idosos oferece vantagens em termos de reabilitação protética dentária, pois esses pacientes requerem uma atenção mais cuidadosa.
\end{abstract}

Palavras-chave: Implantes dentários; Odontologia geriátrica; Prótese dentária fixada por implante.

\begin{abstract}
The objectives of this study were to evaluate implant survival and success rates in elderly patient and investigate the functional and nutritional conditions of the patient after rehabilitation treatment with a protocol-type prosthesis using mediate loading. Anterior extraction of teeth \#33 to \#44 was performed and after six months five Cone-Morse implants of $3.5 \times 11 \mathrm{~mm}$ were placed. The prosthesis installation was completed after a 6-month osseointegration period and followed up for two years. There were no loss of implants, clinical signs of inflammation or presence of bone loss around the implants, and the prosthetic components were perfectly adapted. Thus, the mediate loading protocol in very elderly patients provides advantages in terms of dental prosthetic rehabilitation, as these patients require a more careful attention.
\end{abstract}

Keywords: Dental implants; Geriatric dentistry; Dental prosthesis implant-supported.

\section{Resumen}

El objetivo de este estudio fue evaluar la supervivencia y tasa de éxito de los implantes en pacientes ancianos e investigar las condiciones funcionales y nutricionales del paciente después del tratamiento rehabilitador con una prótesis tipo protocolo, utilizando carga mediada. Se realizaron extracciones de 33 a 44 dientes y a los seis meses se colocaron cinco implantes Cone-Morse de 3,5 x $11 \mathrm{~mm}$. La instalación de la prótesis se completó a los 6 meses de la osteointegración y el seguimiento se realizó durante dos años. No hubo pérdida de implantes, signos clínicos de inflamación o presencia de pérdida ósea alrededor de los implantes, y los componentes protésicos se adaptaron 
perfectamente. Así, el protocolo de carga mediada en pacientes muy ancianos ofrece ventajas en cuanto a la rehabilitación protésica dental, ya que estos pacientes requieren una atención más cuidadosa.

Palabras clave: Implantes dentales; Odontología geriátrica; Prótesis dental de soporte implantado.

\section{Introdução}

De acordo com o Instituto Brasileiro de Geografia e Estatística (IBGE) o Brasil apresenta hoje 26 milhões de pessoas acima dos 60 anos e a estimativa é que este número aumente (Campostrini, 2004). Com o envelhecimento ocorrem modificações no organismo, dentre elas a perda de dentes. Para o indivíduo, este fato pode ser considerado uma mutilação, trazendo um grande impacto para a vida, afetando o relacionamento social, a estética, a fonética e a capacidade mastigatória. Com a dificuldade mastigatória estabelecida, a condição nutricional do paciente pode ser afetada comprometendo a saúde geral (Banu et al., 2016).

Pacientes idosos podem apresentar deficiências nutricionais, além de diferentes comorbidades como doenças sistêmicas e consequentemente o uso de diversas medicações, podendo ser considerado como um paciente especial. O Cirurgião-dentista deve ser capaz de realizar uma anamnese detalhada, a fim de oferecer um tratamento mais seguro. Devido as condições médicas prévias, em alguns casos torna-se rotineiro e mandatório uma consulta ao médico para esclarecimento e orientações sobre a condição sistêmica do mesmo (Banu et al., 2016; Müller et al., 2013).

Geralmente os procedimentos cirúrgicos realizados no consultório odontológico, para exodontias e instalação de implantes, são de curta duração e realizados sob anestesia local. É importante que o paciente esteja estável psicologicamente e que consiga ter uma higiene bucal satisfatória, fator importante para o sucesso e sobrevivência do tratamento (Fernandes et al., 2013).

As próteses de tipo protocolo surgiram com o objetivo de devolver a função aos pacientes que apresentavam bastante dificuldade em se adaptar às próteses totais convencionais, principalmente àquelas colocadas na região de mandíbula severamente reabsorvidas. Este tipo de prótese consiste na colocação de 4 a 6 implantes na região anterior de mandíbula, entre os forames mentuais, e após um período de espera de dois a seis meses, podem sustentar uma prótese fixa com estrutura metálica e dentes em resina acrílica (Adell et al., 1981; Rocha et al., 2013).

De acordo com a literatura, a idade (população geriátrica) não parece ser um fator para o sucesso para instalação dos implantes (Baat, 2000; Engfors et al., 2004; Lee et al., 2010). Doenças sistemáticas que estão geralmente associadas à pacientes idosos, como diabetes e osteoporose podem ser uma contraindicação relativa devido à função imunológica comprometida e à falta de densidade óssea. Outro fator de risco relacionado à longevidade dos implantes dentários é a presença da inflamação nos tecidos peri-implantares (Wen et al., 2014). Alguns estudos mostram que a idade tem relação com a condição peri-implantar dos implantes e com a perda óssea progressiva ao redor dos mesmos (Hoeksema et al., 2015). Em contrapartida, outros estudos revelam resultados contraditórios (Zarb e Schmitt, 1994; Grant e Kraut, 2007).

Portanto, devido a literatura ser limitada em relação à implantodontia na população geriátrica, este estudo teve como objetivo descrever por meio de relato de caso (Pereira et al., 2018; Yin, 2015; Ludke \& Andre et al., 2013; Koche, 2011), a reabilitação com implantes em mandíbula de um paciente idoso com 94 anos de idade, utilizando prótese sob implantes tipo protocolo e avaliar a sobrevivência e o sucesso dos implantes, assim como as condições funcionais e nutricionais do paciente após o tratamento de reabilitação com prótese tipo protocolo mediato.

\section{Metodologia}

Trata-se de um estudo de relato de caso de reabilitação dentogengival sobre implantes em paciente geriátrico com um acompanhamento de 2 anos. Esse estudo apresenta termo de consentimento livre e esclarecido assinado pelo paciente, para a 
participação nessa pesquisa. Todos os princípios éticos da declaração de Helsinque foram respeitados.

\section{Relato de Caso}

Paciente NBC, 94 anos, cor branca, apresentou-se em consultório particular na cidade de Santos - São Paulo, Brasil, para a reabilitação da maxila e mandíbula atrófica. Durante a anamnese foi relatado que o paciente apresentava disfunção arterial, coronária e hipertensão e fazia uso dos medicamentos: AAS 100mg, Trezor $20 \mathrm{mg}$ (Aché®), Vastarel MR $35 \mathrm{mg}$ (Servier Do Brasil $\left.{ }^{\circledR}\right)$, Concor 2,5 mg (Merch $\left.{ }^{\circledR}\right)$, Zyloric 100mg (Aspen Pharma ${ }^{\circledR}$ ) e Lasix $40 \mathrm{mg}\left(\right.$ Sanofi $\left.{ }^{\circledR}\right)$ para controle das disfunções arterial e coronária.

$\mathrm{Na}$ avaliação radiográfica mandibular, foi notado que o paciente apresentava pouca espessura óssea na região anterior e pouca altura óssea na região posterior esquerda (Figura 1 e 2). Porém, tais características não contraindicavam o procedimento de instalação dos implantes. Na avaliação clínica, as próteses totais superior e inferior apresentavam-se em bom estado. Em seguida, foram solicitados exames laboratoriais pré-operatórios (hemograma completo, coagulograma e contagem de plaquetas, uréia e creatinina). Os resultados dos exames estavam dentro dos valores de referência, comprovando o estado de saúde estável do paciente. Antes da realização do procedimento cirúrgico de exodontia, o paciente realizou uma consulta de rotina com o cardiologista, que suspendeu o uso do AAS 7 dias antes da cirurgia para as exodontias e 7 dias antes da cirurgia para colocação de implantes.

Figura 1: Tomografia computadorizada inicial - Imagem 3D pré-operatória evidenciando a condição de mandíbula atrófica.

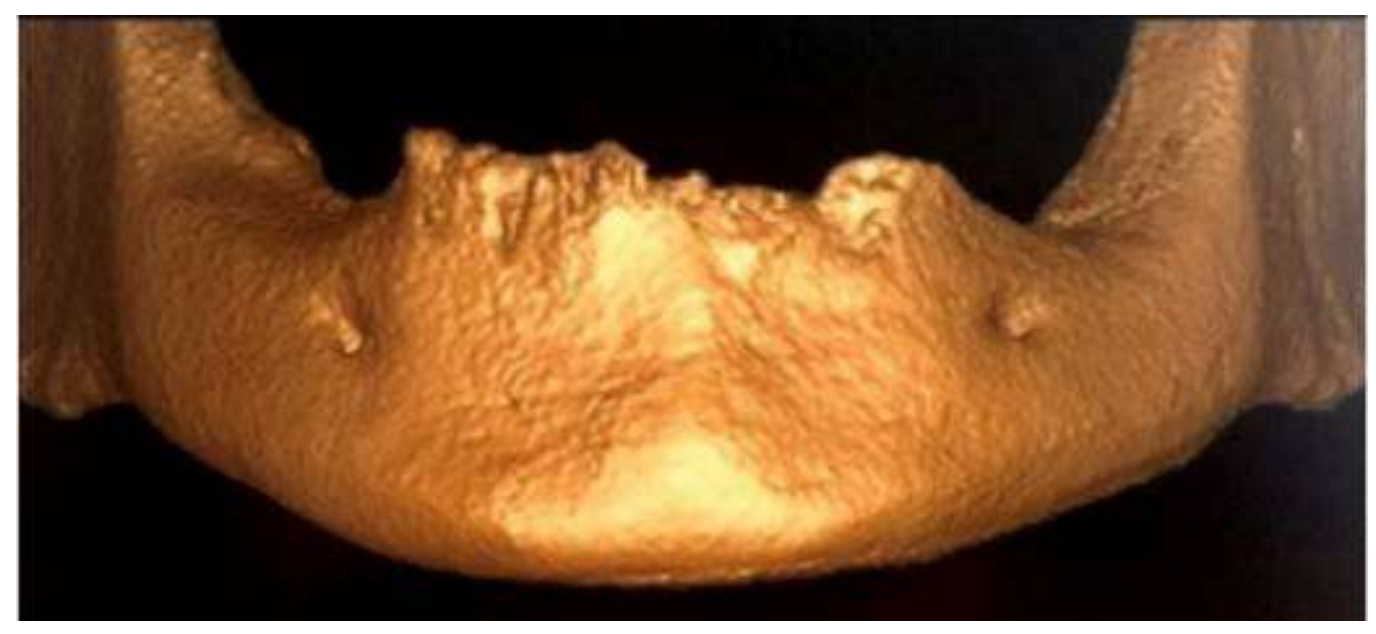

Fonte: Autores.

Podemos observar na Figura 1, imagem em reconstrução 3D de tomografia computadorizada pré-operatória, evidenciando pouca espessura óssea em região anterior de mandíbula. 
Figura 2: Radiografia panorâmica da mandíbula pré-operatória.

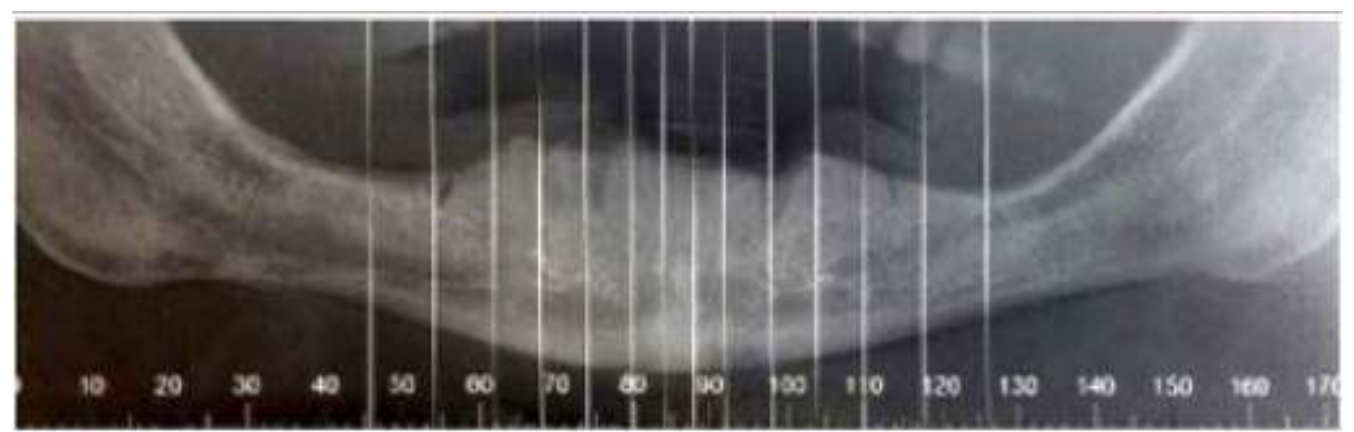

Fonte: Autores.

Na Figura 2, imagem da radiografia panorâmica pré-operatória, em que se evidencia pouca altura óssea em região posterior de mandíbula.

O paciente foi medicado com duas cápsulas de Amoxiclina - $500 \mathrm{mg}$ (EMS®) e um comprimido de Decadron - $4 \mathrm{mg}$ (Aché®), uma hora antes da cirurgia. Ao iniciar o procedimento para realizar a exodontia dos dentes 33 ao 44, foi aplicado anestesia infiltrativa bilateralmente na região do nervo mentual, nervo incisivo e ligamento periodontal dos dentes a serem extraídos com anestésico local Mepivacaína 2\% com Epinefrina 1:100.000 (Mepiadre, DFL®). Após a exodontia, foi realizada sutura simples dos alvéolos. Como medicação pós-operatória foi receitado uma cápsula de Amoxacilina $500 \mathrm{mg}$ a cada 8 horas durante 7 dias e um comprimido de $1 \mathrm{~g}$ de dipirona a cada 8 horas durante 2 dias.

Após 6 meses foi realizada uma nova cirurgia para colocação dos implantes. Antes do procedimento cirúrgico, a prótese total inferior foi duplicada em resina acrílica para que fosse utilizada como guia cirúrgico para a instalação de 5 implantes em mandíbula.

O mesmo protocolo cirúrgico foi executado nesta cirurgia. Foi realizada uma incisão mucoperiostal e descolamento do retalho e foram inseridos 5 implantes 3.5 x $11 \mathrm{~mm}$, cone Morse (Neodent - Sistema de Implantes - Titamax CM®), um na região central, dois mesiais e dois distais entre os forames mentuais (Figura 3). Após a instalação dos implantes e colocação de parafuso de cobertura de $2.0 \mathrm{~mm}$, foi realizada a sutura com fio de nylon (Ethicon 5.0). A medicação pós-operatória foi a mesma realizada no procedimento cirúrgico anterior. Após 6 meses da inserção dos implantes, foi realizada a reabertura e colocação de cicatrizadores, todos com $2,5 \mathrm{~mm}$ e com perfil de $3.3 \mathrm{~mm}$. 
Figura 3: Tomografia computadorizada de feixe cônico, corte axial após a cirurgia de instalação dos implantes evidenciando o posicionamento tridimensional.

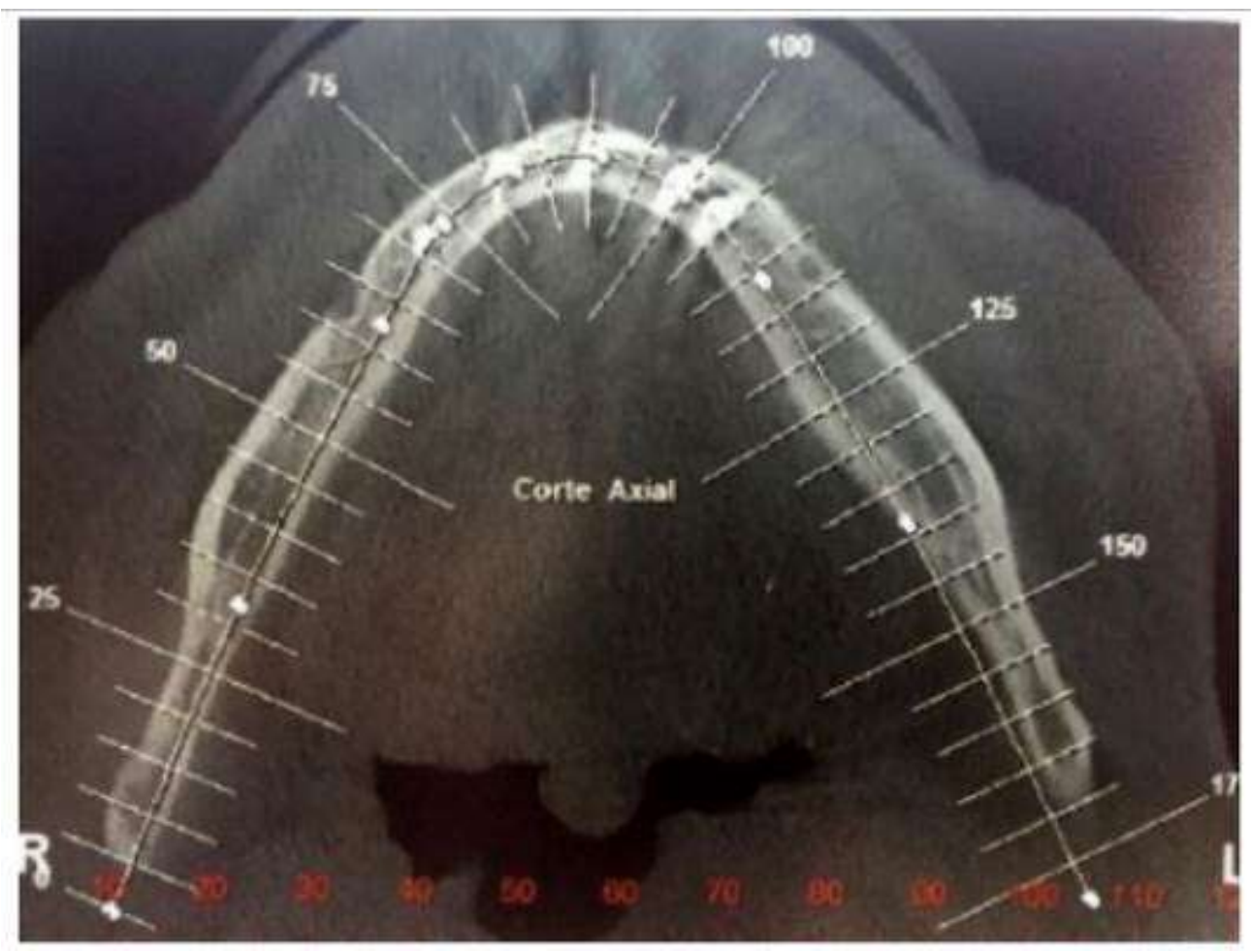

Fonte: Autores.

$\mathrm{Na}$ imagem acima podemos observar a tomografia pós-operatória, em corte axial, evidenciando o local de inserção dos 5 implantes em mandíbula, um na região central, dois mesiais e dois distais entre os forames mentuais.

Após uma semana, mini pilares foram instalados com 32 N.cm, de acordo com a recomendação do fabricante, sendo os 5 mini-pilares cônicos retos com transmucoso de 1,5 mm. Em seguida, foi realizada a moldagem de transferência dos pilares com moldeira aberta, utilizando silicone de adição Futura AD (DFL $®$, Rio de Janeiro, Brasil), em fase única (fluida + pesada) (Figura 4). Sobre o modelo adquirido, coifas foram confeccionados e uma base de prova com rolete de cera para o registro de mordida e dimensão de oclusão. Uma nova prova da prótese foi feita, já sobre a estrutura metálica confeccionada com dentes, com a dimensão adquirida anteriormente, tomando-se o cuidado de fixar o conjunto por todos os 5 parafusos sobre os minipilares, aumentando a estabilização do conjunto. Após a prova dos dentes, foi realizada a inclusão em mufla e acrilização da peça seguida pelo acabamento, polimento e ajustes oclusais em boca no momento da entrega da prótese. 
Research, Society and Development, v. 10, n. 3, e52710312972, 2021

(CC BY 4.0) | ISSN 2525-3409 | DOI: http://dx.doi.org/10.33448/rsd-v10i3.12972

Figura 4: Análogo dos implantes no modelo de gesso.

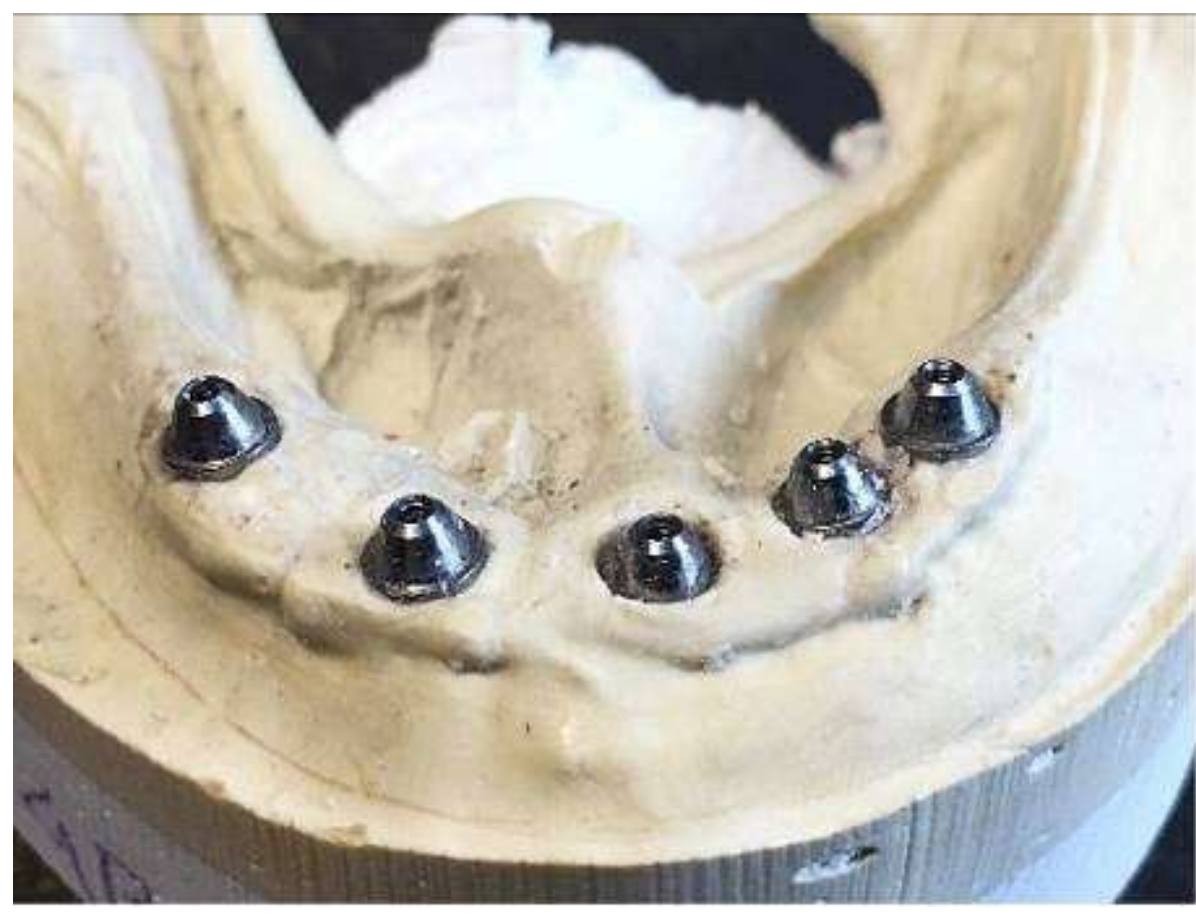

Fonte: Autores.

Na imagem acima observa-se o modelo de transferência, com a posição exata dos pilares protéticos em mandíbula, possibilitando a confecção da prótese.

Após o acompanhamento de 2 anos, não houve perda óssea ao redor dos implantes (Figura 5), não houve presença de inflamação no tecido gengival e não foi observada nenhuma alteração na adaptação da prótese (Figura 6). Sendo assim, foram preservadas as funções estéticas, mastigatória e fonética.

Figura 5: Radiografia panorâmica de acompanhamento após 2 anos da instalação dos implantes.

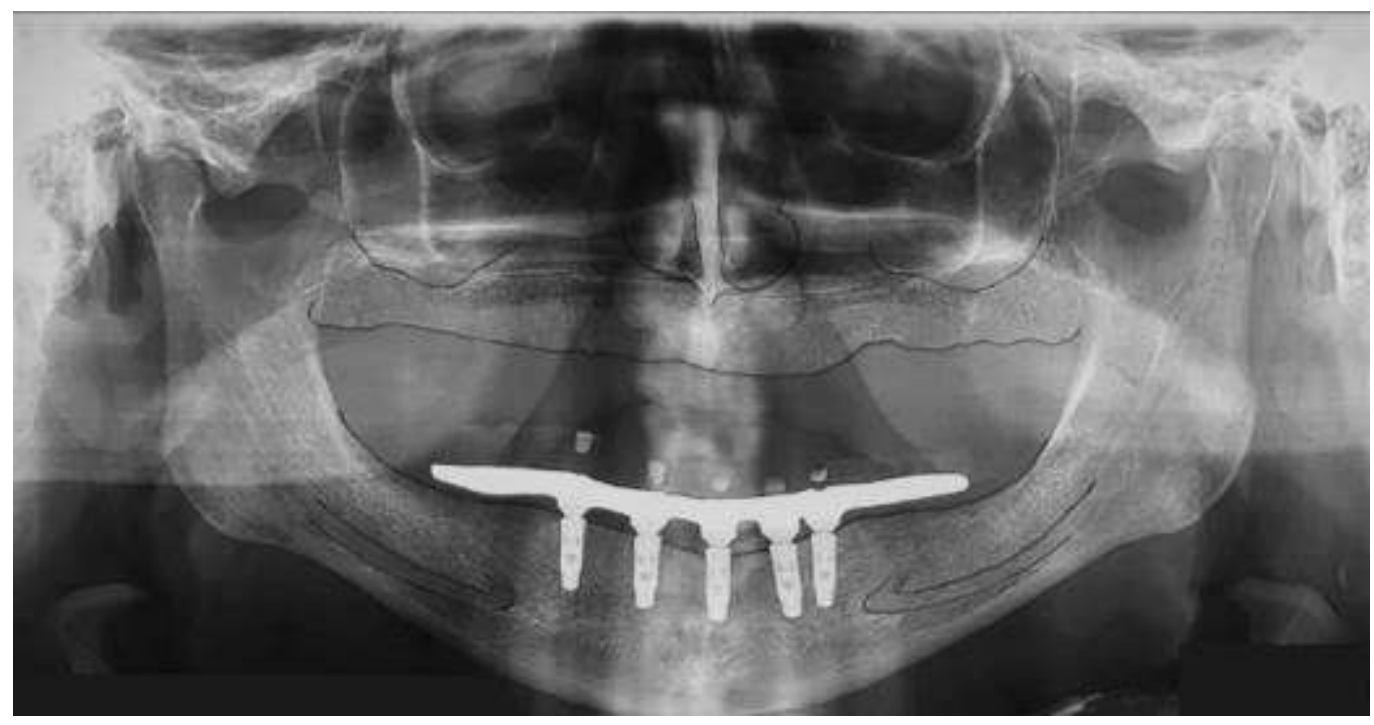

Fonte: Autores. 
Nota-se na radiografia panorâmica acima, que após 2 anos da instalação dos implantes, não houve perda óssea ao redor dos implantes.

Figura 6: Dois anos de acompanhamento da instalação da prótese tipo protocolo - vista frontal.

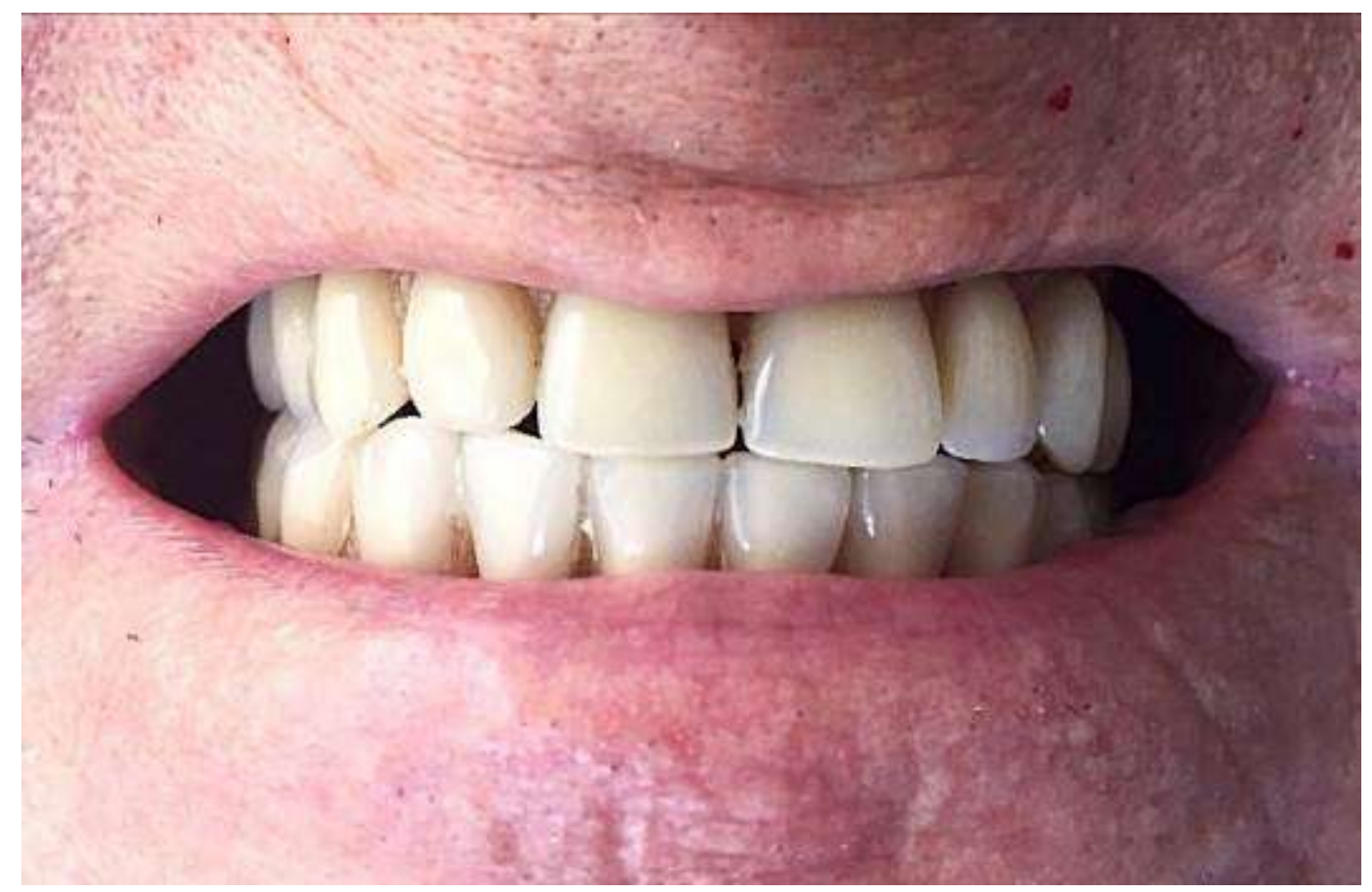

Fonte: Autores.

A imagem acima é referente há 2 anos de acompanhamento. Podemos observar a prótese bem adaptada, reestabelecendo função e estética para o paciente.

\section{Discussão}

O objetivo do presente estudo foi relatar um caso de reabilitação de mandíbula com prótese protocolo (carga mediata), em paciente com idade avançada (94 anos), desdentado superior e inferior, a fim de avaliar a sobrevivência e o sucesso dos implantes e as condições funcionais e nutricionais do paciente após 2 anos de acompanhamento.

Embora não sendo considerada uma regra geral, o edentulismo está frequentemente associado ao paciente geriátrico (Müller et al., 2013). A reabilitação por meio de prótese do tipo protocolo, mesmo em 2 tempos cirúrgicos, foi a prótese escolhida devido à idade do paciente, a fim do mesmo suportar o tempo de trabalho exigido para a sua reabilitação e diminuir o risco de perda dos implantes (Grant e Kraut, 2007).

A taxa de sucesso das próteses implanto-suportadas é variável, porém quando comparada aos métodos tradicionais de reposição dentária, tem maior longevidade e melhoria na função, preservação óssea e melhores resultados psicológicos (Compton et al., 2017). Esses achados são compatíveis com este relato de caso, pois os implantes não apresentam perda óssea após 2 anos de acompanhamento.

Neste relato de caso, poderia ter ocorrido a falha dos implantes dentais e da prótese devido a idade avançada do paciente. Porém, não houve perda de implantes, sinais clínicos de inflamação, presença de perda óssea ao redor dos implantes e os componentes protéticos apresentavam-se em perfeita adaptação. Um dos fatores atribuídos ao sucesso do caso foi a manutenção tecidual realizada a cada seis meses e o cuidado do paciente quanto a higienização da sua prótese. 
A estabilidade e retenção de uma prótese implanto-suportada são melhores quando comparadas às próteses mucosuportadas. A fonética de uma prótese tradicional pode ser prejudicada pela instabilidade da mesma (Müller et al., 2013). Pacientes tratados com prótese implanto-suportadas, relatam que a condição psicológica melhorou em torno de $80 \%$ quando comparada ao estado prévio, ou seja, enquanto usavam prótese removível ou muco-suportada (Misch et al., 2014).

Baseado no relato do caso, a instalação de prótese do tipo protocolo em dois tempos (carga mediata), foi a opção de tratamento mais adequada, já que se trata de um paciente geriátrico. Embora, o estado geral de saúde do paciente fosse estável, ele apresentou dificuldades motoras durante o tratamento e algumas outras características peculiares do processo de envelhecimento. Essas características foram consideradas durante o tratamento com o objetivo de aumentar o grau de satisfação pessoal, a melhoria da autoestima, reestabelecer função e proporcionar um aspecto facial mais harmônico e agradável através da reabilitação oral. Para esses pacientes é importante também estabelecer um rígido controle e manutenção periódica para garantir o sucesso do tratamento.

\section{Conclusão}

A reabilitação oral com prótese tipo protocolo carga mediata demonstrou bons resultados e é uma opção de tratamento viável para pacientes geriátricos, uma vez que esses pacientes necessitam de cuidados especiais com a saúde geral, além de atendimento odontológico individualizado. Trabalhos futuros devem ser realizados com objetivo de verificar a longo prazo, a resposta dos tecidos peri-implantares diante uma prótese dentogengival.

\section{Referências}

Adell, R., Lekholm, U., Rockler, B. \& Branemark, P-I. (1981) A 15-year study of osseointegrated implants in the treatment of the edentulous jaw. Int J Oral Surg, 6, 387-416.

Baat, C. (2000). Success of dental implants in elderly people - a literature review. Gerodontology, 17, 45-48.

Banu, R. F., Veeravalli, P. T. \& Kumar, V. A. (2016). Comparative Evaluation of Changes in Brain Activity and Cognitive Function of Edentulous Patients, with Dentures and Two-Implant Supported Mandibular Overdenture-Pilot Study. Clin Implant Dent Relat Res, 3, $580-7$.

Campostrini, E. (2004). Aspectos Sociais e familiares do envelhecimento. In: Campostrini E, Odontogeriatria. Livraria e Editora Revinter Ltda, 28 -31.

Compton, S. M., Clark, D., Chan, S., Kuc, I., Wubie, B. A. \& Levin, L. (2017). Dental Implants in the Elderly Population: A Long-Term Follow-up. Int J Oral Maxillofac Implants, 1, 164-170.

Engfors, I., Ortorp, A. \& Jemt, T. (2004). Fixed implant supported prostheses in elderly patients: A 5-year retrospective study of 133 edentulous patients older than 79 years. Clin Implant Dent Relat Res, 6, 190-198.

Fernandes Jr, V. V. B., Maciel Jr, M. S., Feitosa, P. C., Rodrigues, R. M., Zamboni, E. \& Romeiro, R. L. (2013). Protocolo Imediato Provisório - relato de caso. Full Dentistry in Science, 4, 277-281.

Grant, B. T., Kraut, R. A. (2007). Dental implants in geriatric patients: a retrospective study of 47 cases. Implant Dent, $4,362-8$.

Hoeksema, A. R., Vissink, A., Peters, L. L., Meijer, H. J., Raghoebar, G. M. \& Visser, A. (2015). Peri-implant health in people aged 75 and over with an implant-retained overdenture in the mandibula. Ned Tijdschr Tandheelkd, 122, 383-390.

Koche, J. C. (2011). Fundamentos de metodologia científica. Vozes.

Lee, H. J., Kim, Y. K., Park, J. Y., Kim, M. J. \& Yun, P. Y. (2010). Short-term clinical retrospective study of implants in geriatric patients older than 70years. Oral Surg Oral Med Pathol Oral Radiol Endod, 110, 442-446.

Ludke, M. \& Andre, M. E. D. A. (2013). Pesquisas em educação: uma abordagem qualitativa. E.P.U.

Misch, C. E. (2014). Dental Implant Prosthetics. Elsevier -Health Sciences Division.

Müller, F., Duvernay, E., Loup, A., Vazquez, L., Herrmann, F. R. \& Schimmel, M. (2013). Implant-supported mandibular overdentures in very old adults: a randomized controlled trial. J Dent Res, 12, 154S-60S.

Pereira, A. S. et al (2018). Metodologia da pesquisa científica. UFSM.

Rocha, S. S., Souza, D. R., Fernandes, J. M. A., Garcia, R. R. \& Zavanelli, R. A. (2013). Próteses Totais Fixas Tipo Protocolo Bimaxilares. Relato de Caso. Rev Odontol Bras Central, 60, 21-27. 
Research, Society and Development, v. 10, n. 3, e52710312972, 2021

(CC BY 4.0) | ISSN 2525-3409 | DOI: http://dx.doi.org/10.33448/rsd-v10i3.12972

Wen, X., Liu, R., Li, G., Deng, M., Liu, L., Zeng, X. T. \& Nie, X. (2014). History of periodontitis as a risk factor for long-term survival od dental implants: A meta-analysis. Int J Oral Maxillofac Implants. 29, 1271-1280.

Yin, R. K. (2015). O estudo de caso. Bookman.

Zarb, G. A. \& Schmitt, A. (1994). Osseointegration for elderly patients: The Toronto study. J Prosthet Dent, 72, 559-568. 